\title{
Desarrollo de la viticultura y servicios para la producción en el valle de Copiapó (Chile) en el contexto de la mundialización económica ${ }^{1}$
}

\author{
Severino Escolano Utrilla² y Jorge Ortiz Véliz ${ }^{3}$
}

\begin{abstract}
RESUMEN
Los procesos actuales de reestructuración territorial se relacionan con las nuevas formas técnicas y de organización de la producción, especialmente con la integración de los flujos de personas, bienes, servicios, capitales e información en un sistema mundial, y con la internalización de las empresas. En Chile, desde 1970, se ha producido un movimiento para incorporar a la sociedad y economía nacionales en los sistemas mundiales. Algunas regiones se han especializado en producciones mineras y agrarias orientadas a la exportación, como el valle de Copiapó en Atacama. Esta conexión ha reactivado la actividad económica y promovido intensos cambios en la sociedad y el territorio de la zona. Sin embargo, la diversificación de las funciones urbanas de Copiapó no se ha producido en la misma proporción de las magnitudes económicas de la región. Si bien los servicios comerciales de apoyo inmediato se han incrementado, la mayor parte de los avanzados se prestan desde otras ciudades.
\end{abstract}

Palabras clave: Globalización, servicios para la producción, viticultura, economía local, Copiapó.

\begin{abstract}
The current processes of territorial restructuration is related with new technique forms and organization of production, especially with the integration of people flows, goods, services, capitals, and world system information; and with the internationalization of companies. Since 1970 there has been in Chile a movement in order to incorporate the society and national economy inside the world systems. Some Chilean regions, like Copiapo Valley in Atacama, have specialized in mineral an agricultural production orientated to exportation. This connection has reactivated the activity economically and promoted many changes inside society and throughout the territory of the zone. Nevertheless, the diversification of Copiapo's urban functions hasn't had the same economic magnitudes than the rest of the region. Even though commercial services of immediate support have increased, most of advanced ones are provided from other cities.
\end{abstract}

Key words: Globalization, producer services, wine growing, local economics, Copiapó.

1 Proyectos FONDECYT No 1071102 y No 7080082. Artículo recibido el 14 de septiembre de 2009 y aceptado el 16 de marzo de 2010.

2 Departamento de Geografía y Ordenación del Te- rritorio, Universidad de Zaragoza (España). E-mail: severino@unizar.es

3 Departamento de Geografía, Universidad de Chile (Chile).E-mail: jortiz@uchile.cl 
Una de las cualidades esenciales del proceso de reorganización general de las sociedades actuales se define por la creciente tendencia hacia la integración de los flujos financieros, de información, de personas y bienes, en un sistema mundial de producción y consumo, cuyos efectos alcanzan tanto a cada una de las dimensiones de la vida social, como a los componentes de la organización territorial a todas las escalas.

En Chile, desde mediados de la década de 1970, los sucesivos gobiernos han tomado decisiones encaminadas a incorporar a la sociedad y economía nacionales en los sistemas mundiales. De forma paulatina, se han puesto en marcha nuevas medidas reguladoras de la relación capital-trabajo, se ha liberalizado la circulación de capitales, se han privatizado servicios públicos, en especial los urbanos, y se han promulgado otras disposiciones que redefinen la función del Estado en la producción y reproducción social y, por supuesto, su cometido en la planificación y ordenación territorial. Así pues, todas las transformaciones territoriales recientes se desarrollan en un nuevo marco socioeconómico e ideológico de acumulación flexible bastante consolidado.

Esta actuación consistente de políticas neoliberales durante las tres últimas décadas ha gestado un temprano modelo socioeconómico específico que difiere, en diversos aspectos, del de otros países vecinos (Velut, 2005). De manera simple, se puede afirmar que el modelo chileno se caracteriza por su elevado grado de liberalización, tal vez el más alto entre los países latinoamericanos, pero también por el considerable crecimiento de algunas magnitudes macroeconómicas.

Son contadas las partes de la sociedad, del territorio y la economía chilenos que escapan a la influencia directa o indirecta de este proceso. En el haber del mismo se suelen anotar las espectaculares tasas de crecimiento económico del Producto Interno Bruto, la subida de la renta per cápita, o la modernización de varios sectores productivos y de los servicios. Entre 1975 y 1979 el Producto Interno Bruto creció alrededor del 7\% anual (De Mattos et al., 2004), en 2001 lo hizo al $4,1 \%$ y en 2006 al $4,3 \%$ (INE, 2009). Pero los efectos negativos son también aplastantes y patentes, y van desde la fuerte dependencia de la economía nacional de las coyunturas de la economía mundial, hasta el notorio incremento de las desigualdades sociales y de las disparidades regionales de la renta, pasando por el galopante deterioro del medio ambiente y la depredación de los recursos naturales, entre otros.

La acción conjunta de los elementos apuntados y otros impulsa, y a la vez opera como su marco, un movimiento de reestructuración territorial a varias escalas, cuya dinámica difiere de la etapa anterior. En efecto, la concepción y práctica dominantes arribaabajo de la política territorial, en cuya cúspide se sitúa el estado central, es alterada por la aparición de nuevos y poderosos agentes internacionales, por una parte, y regionales y locales, por otra. La interacción, conflictiva o de colaboración, entre todos estos agentes, junto con las condiciones ecológicas locales, da lugar a procesos complejos de reorganización territorial que modifican el statu quo regional previo: algunas áreas o regiones avanzan mientras que otras se estancan o retroceden.

Desde la publicación de la obra de Benko y Lipietz (1994), es frecuente utilizar esta clasificación dicotómica para caracterizar la evolución general de las regiones en relación con su competitividad en los mercados internacionales. No obstante, como señalan Szary (1997) y Gónzález y Rodríguez (2006), conviene matizar los aspectos y dimensiones e incluso los espacios intrarregionales en los que las regiones ganan o pierden. En este sentido, este artículo contribuye a precisar los procesos y alcance de los impactos de la mundialización económica en el espacio estudiado.

La mayoría de los estudios realizados sobre los impactos territoriales de la globalización se refieren a las transformaciones sociales, urbanísticas, económicas y espaciales de las grandes ciudades, y se han centrado casi en forma exclusiva en el Gran Santiago. Sin embargo, apenas se ha investigado sobre el tema en otros espacios, en particular aquellos especializados en producciones agrarias, silvícolas o mineras, a pesar de la reconocida revalorización del territorio como "ámbito privilegiado para el análisis de los procesos económico-sociales" (Riffo y Calisto, 1998: 53). 
En el contexto delimitado, en este artículo se aborda el estudio de algunas transformaciones territoriales en el valle del Copiapó (Región de Atacama) asociadas a la expansión de la viticultura orientada a la exportación, y el desarrollo de los servicios a la producción en la ciudad de Copiapó. Se considera que es importante conocer las relaciones que existen entre la producción agrícola especializada y orientada a la exportación, y la expansión de los servicios a las empresas, ya que el conocimiento de los nexos entre conglomerados de actividad económica local es fundamental para el diseño y aplicación de nuevas políticas territoriales.

\section{Objetivos y marco teórico: desarrollo y localización de los servicios a las empresas}

Desde las primeras interpretaciones científicas del régimen de acumulación flexible se ha otorgado una función crucial a la tríada indisociable de categorías socioeconómicas servicios-tecnología-información, no solo como componentes y motores del capitalismo moderno, sino también como factores de transformación territorial (Daniels, 1985; Illeris, 1985; Gershuny y Miles, 1988; Albrechts et al., 1989; Illeris, 1989; Daniels, 1991; Coffey \& Bailly, 1992; Moreno y Escolano, 1992b; Daniels, 1993; Moulaert y Scott, 1997).

La creciente importancia de los servicios en las sociedades modernas se manifiesta en su elevada participación en el valor de la producción (de los países y regiones), en el gran volumen de ocupados en el sector y su fuerte participación en el desenvolvimiento de la vida social y de los individuos. Esta pujanza se explica, entre otras razones, por el galopante crecimiento de la demanda de los individuos, de las empresas y de las instituciones, por la renovación de los métodos y organización de la producción, por la actuación de las innovaciones tecnológicas, y por la reestructuración mundial de algunos mercados (Daniels, 1985; Noyelle, 1986; Illeris, 1989; Rada, 1989)

Por eso, para valorar debidamente la naturaleza y el alcance de los servicios en la sociedad, en la economía y en el territorio, es necesario tener en cuenta su carácter estraté- gico en la producción de bienes (y servicios). En efecto, la imbricación de los servicios y las actividades de producción ha generado un único proceso productivo bienes-servicios, integrado y continuo (Barcet et al., 1984; Bailly y Coffey, 1994).

Dentro del sector servicios, los servicios a las empresas constituyen un grupo de especial relevancia. Su carácter diferenciador reside en su orientación hacia mercados intermedios, es decir, el resultado de su actividad se dirige no al mercado final, sino principalmente a otras empresas o instituciones (agrarias, industriales o de servicios). En definitiva: su output se incorpora como insumo en el proceso productivo, bien en el seno de la misma compañía, o es adquirido en el mercado.

Esta conexión directa con las unidades de producción convierte a este tipo de servicios, y especialmente a los servicios avanzados a la producción (investigación, seguros, asesoría jurídica, auditoría, entre otros) en un resorte imprescindible para mejorar la competitividad de las empresas, a la vez que acrecienta su trascendencia territorial (Daniels, 1985; Bailly y Maillat, 1989; Daniels y Moulaert, 1991; Moreno y Escolano, 1992a).

Uno de los objetivos más importantes del estudio de esta clase de servicios ha consistido en explicar y modelar sus pautas de localización (Moulaert y Gallouj, 1993; Beaverstock et al., 1999). En general, los servicios a las empresas siguen diversas lógicas espaciales que varían entre la vecindad a las unidades de producción, y la concentración en ciudades de rango elevado en la jerarquía urbana. Precisamente, la abundancia y diversidad de los servicios a las empresas se utiliza como indicador básico para determinar el rango y la función de las ciudades en las redes urbanas. Estas tendencias son bien conocidas desde hace tiempo, y se relacionan principalmente con la naturaleza del servicio producido y con la tecnología utilizada; numerosas investigaciones han confirmado la dualidad dispersión-concentración de la organización territorial de los servicios a la producción (Martinelli, 1991b; Monnoyer y Philippe, 1991; Parnreiter et al., 2007).

En efecto, varias actividades de servicio a los productores, especialmente las de carác- 
ter avanzado, hacen uso intensivo de información y de profesionales muy cualificados. Por otra parte, las nuevas tecnologías de la información permiten la organización de las empresas en redes mundiales, regionales o locales, lo que facilita la división espacial del trabajo y su localización en grandes ciudades. En estas últimas, se encuentran también las infraestructuras tecnológicas y los conocimientos necesarios para las transmisión y tratamiento de la información digital, las principales instituciones y otras empresas y servicios que posibilitan el desarrollo de relaciones interpersonales y el disfrute del tiempo de ocio, todo lo cual configura un medio adecuado para la instalación de servicios avanzados a los productores (Coffey y Polese, 1987; Sassen, 1991; Moreno y Escolano, 1992a; Daniels, 1993; Moulaert et al., 1997; Sassen, 2003). La separación física de las empresas de servicios a los productores de las unidades de producción, posibilita el control remoto de parte de las economías locales y regionales por compañías ubicadas en grandes ciudades (Bailly, 1985).

En este artículo se exploran las relaciones entre la expansión de la viticultura y el crecimiento de los servicios a la producción agraria en el marco general expuesto del desarrollo de los servicios a los productores. Sus contenidos se estructuran en torno a dos líneas de indagación principales consistentes en:

1. Identificar algunas características del modo de expansión de la viticultura en el valle de Copiapó y argumentar sus posibles repercusiones en la economía y sociedad locales, en particular en el sector de los servicios a la producción en la ciudad de Copiapó.

2. Explorar y valorar el crecimiento de los servicios a la producción asociados a la actividad agrícola.

Los anteriores enunciados de investigación están guiados por las siguientes hipótesis:

- La creación de nuevas empresas agrícolas, sobre todo en el caso de producciones orientadas a la exportación, es muy probable que conlleve el nacimiento de servicios a los productores.
- En general, las empresas de servicios a los productores se localizan en las ciudades, pero según la naturaleza del servicio y las dimensiones y formas de organización de las empresas que lo producen, aquellas pueden ubicarse en ciudades próximas, o bien en otras a veces muy alejadas de los lugares de producción. Para el caso de Chile, se ha comprobado que la mayor parte de las funciones de "producción inmaterial" (entre ellas los servicios avanzados a la producción) se localizan en Santiago (Escolano et al., 2007). Otros servicios a los productores, como los de baja especialización, pueden encontrarse incluso en asentamientos de pequeño tamaño demográfico.

El recorrido espiral de la relación servicios-ciudad completa un giro, ya que la creación de nuevas empresas (de servicios, o de producción) modifica la estructura física de los tejidos urbanos, la posición y funciones en la jerarquía urbana, así como las bases territoriales y los mercados de trabajo. Estas circunstancias crean nuevos problemas para las instituciones locales, que generan renovadas estrategias de planeamiento, que a su vez cambian las condiciones para la instalación de nuevas empresas.

\section{Metodología y datos}

Para aprender las principales dimensiones estudiadas en este trabajo, se han combinado dos tipos de información obtenida por vías diferentes, y cuya naturaleza y alcance son también distintos.

Por una parte, se han realizado entrevistas semiestructuradas a gerentes y personal técnico de explotaciones agrícolas del valle de Copiapó, así como a técnicos de planeamiento, y empresarios de comercio y hotelería. Esta campaña, realizada en junio y septiembre de 2007, ha permitido conocer las opiniones de estos agentes sociales acerca de la situación y problemas de las empresas, así como el contexto socioeconómico local y los modos de operación de las empresas. Además, estas instancias se aprovecharon para observar sobre el terreno el manejo técnico de los cultivos y las explotaciones. 
Por otra, se han utilizado los datos procedentes de censos, encuestas y otras fuentes secundarias. La más importante ha sido el Registro de Patentes de Copiapó, que ha servido para identificar y trazar la evolución de los servicios a las empresas agrarias entre los años 2000 y 2007. Esta información básica se completó con otra procedente de trabajo de campo, como la localización de cada establecimiento de servicios y el estado general del mismo (rotulación, metros de la fachada y otros).

La población ocupada en actividades económicas ha constituido la base de la clasificación funcional de las ciudades. La población ocupada, se ha obtenido a partir de la variable giro del Censo Nacional de Población y Vivienda (INE, 2002) que distingue 60 tipos de actividades económicas más otro para los no clasificados (códigos de actividad económica, revisión 3 a 2 dígitos).

Estas categorías se han utilizado para tipificar el trabajo -las funciones- que realizan las ciudades en la red urbana. Además, se han construido dos nuevas clasificaciones mediante la agrupación de las categorías del giro para reducir su variabilidad: una está compuesta por la agrupación de las 17 ramas de la clasificación nacional de actividades económicas, y la otra por tres esferas de actividad: producción, intermediación y reproducción social (Beckouche \& Damette, 1993), que se adapta mejor a la realidad del sistema productivo que la tradicional división en sectores económicos.

Un paso previo para obtener los datos censales mediante la aplicación REDATAM-G4 ha consistido en establecer una definición operativa de ciudad. Se ha considerado como tal al conjunto de los distritos (entidad espacial del censo) de un municipio calificados como urbanos en el censo, contiguos o no, que suman más de 5.000 habitantes en 2002. Para cada una de las 193 ciudades así delimitadas se ha contabilizado su población en diversos años y los ocupados en cada categoría de la variable giro (se han utilizado datos válidos para 191 ciudades).

La especialización funcional de cada ciudad, y de los grupos de ciudades en relación con el conjunto nacional se ha medido a través del Coeficiente de Localización (QL) cuyos fundamentos se encuentran en la conocida teoría de la base económica; su razón matemática se expresa:

$$
\mathrm{QL}_{\mathrm{ij}}=\left(\mathrm{X}_{\mathrm{ij}} / \mathrm{X}_{\mathrm{i}} \cdot\right) /\left(\mathrm{X}_{\cdot \mathrm{j}} / \mathrm{X} . .\right)
$$

Donde, coeficiente de localización de la actividad $j$ en la ciudad $i\left(\mathrm{QL}_{\mathrm{ij}}\right)$ :

$X_{\mathrm{ij}}$ : ocupados en la actividad $j$ en la ciudad $i$.

$\mathrm{X}_{\mathrm{i}}$ : ocupados en todas las actividades de la ciudad $i$.

$X_{. j}$ : ocupados de la actividad $j$ en todas las ciudades.

$X_{. .}$: valor de la variable $X$ (ocupados) de todas las actividades en todas las ciudades.

Los límites del $\mathrm{QL}$ son $0 \leq \mathrm{QL} \leq \infty$. Los valores superiores a la unidad en una actividad y ciudad determinadas son indicativos de especialización en relación con la intensidad promedio del conjunto de ciudades; por tanto, es probable que toda o parte de la producción de esa actividad se destine a mercados no locales.

\section{El desarrollo de la viticultura: grandes explotaciones, uso intensivo de tecnología y condiciones agroecológicas favorables}

La proyección de la economía chilena en los mercados internacionales es patente, entre otras manifestaciones en la elevada inversión directa de capitales extranjeros en diversas actividades económicas, en el considerable volumen de las exportaciones, o en los acuerdos comerciales multilaterales y bilaterales (Estados Unidos, Unión Europea, Corea del Sur, EFTA, Nueva Zelanda, entre otros) que Chile mantiene para promover "el desarrollo orientado a la exportación" (Barton et al., 2007: 25). Así, a la tradicional exportación de cobre se han sumado otras materias primas y productos derivados de la pesca, agricultura, ganadería y silvicultura que engrosan un nuevo y dinámico sector exportador no tradicional (Arnade \& Sparks, 1993; Gwynne, 1996; Gwynne, 1999). El valor de las exportaciones de este sector se ha multiplicado por 5 entre 1990 y 2008 (2.779 
millones de dólares en 1990; 15.605 millones de dólares en 2008), lo que ha significado el crecimiento de las exportaciones agropecuarias chilenas en el conjunto mundial, que han pasado del $0,7 \%$ en 1990 al $1,2 \%$ en 2008 (Organización Mundial del Comercio, 2009).

La gran capacidad de los productos chilenos para mantenerse en mercados internacionales se debe, básicamente, a su buena calidad y a los precios competitivos. Al margen consideraciones sobre aranceles y comercio internacional, estas cualidades se logran mediante la explotación intensiva del enorme potencial agroecológico para la viticultura de las regiones semiáridas del Norte Chico y centro sur del país, la aplicación de tecnología avanzada y el uso de mano de obra cualificada y barata.

Estas coordenadas delimitan y permiten explicar el gran desarrollo de algunas actividades del sector primario, en particular de la viticultura cuyos productos, el vino y la uva de mesa, se dirigen prioritariamente a mercados externos.

\section{La creciente importancia económica de la agricultura}

En una región como la de Atacama, cuya economía es altamente dependiente de la minería del cobre, el desarrollo de la viticultura cobra renovada importancia como uno de los factores más relevantes de transformación económica, social y territorial de la región, cuya trascendencia se acentúa en el valle de Copiapó. El sector agrario ha incrementado su participación en el Producto Interno Bruto Regional (1985: 8,6\% del PIB regional; 1996: 4,5; 2006: $16,7 \%$ ), y se ha elevado al segundo puesto tras la minería. Su significación es todavía mayor en la proporción de la fuerza de trabajo (alrededor del $12 \%$ regional en 2006 y 2007). La uva de mesa es el principal rubro de las exportaciones agrícolas, ya que en 2004 supuso el 5\% de las exportaciones regionales. Cabe añadir también que las tradicionales actividades hortícolas y olivicultura han modernizado sus explotaciones, de tal forma que sus productos (aceitunas, aceite) contribuyen no solo a diversificar las producciones agrarias regionales sino también a engrosar los rubros de las exportaciones agrarias de la región.
Los viñedos se localizan en los valles transversales de Huasco y, sobre todo, de Copiapó, aprovechando las excepcionales condiciones agroecológicas para el crecimiento de las vides, en especial la abundante insolación y la disponibilidad de recursos hídricos. En el valle de Copiapó, los cultivos se extienden por las partes bajas y las laderas de ambas vertientes del valle, entre los 450 y los 1.500 metros de altitud, a veces en parcelas con pendientes muy fuertes, al límite para el trabajo con maquinaria agrícola (Figura № 1 ). Los suelos desarrollados en climas semiáridos exigen un manejo cuidadoso para su uso agrícola intensivo (alto grado de salinidad, pH básico, entre otras características), lo que se traduce en fuertes inversiones tecnológicas para mantenerlos y evitar su degradación. Los olivares y las explotaciones hortícolas se concentran principalmente aguas abajo de la ciudad de Copiapó.

Por otra parte, el aumento de la extensión de los viñedos (Cuadro $N^{0} 1$ ) y la creciente demanda de agua para otras actividades económicas y para uso doméstico, han presionado fuertemente sobre los recursos hídricos superficiales y subterráneos, de tal manera que estos pueden convertirse en un factor limitante para el crecimiento económico (Gobierno Regional de Atacama, 2005). La mejora de la gestión y de la eficiencia de uso de los recursos hídricos es uno de los ejes estratégicos del Plan de Gobierno de la Región de Atacama 2006-2010 (SERPLAC, 2005).

Finalmente cabe añadir que la situación de la zona en el hemisferio Sur es una ventaja adicional, ya que los productos se venden principalmente en mercados del hemisferio Norte (Estados Unidos, México y Unión Europea).

Como se aprecia en el Cuadro $N^{0} 1$ y en la Figura $N^{\circ} 2$, la mayor parte del viñedo de la región se encuentra en el valle de Copiapó y se dedica a la producción de uva fresca. La evolución de su superficie desde 1994 muestra diferentes trayectorias comunales: en Copiapó apenas varía hasta 2005, que aumenta unas 200 ha, mientras que en Tierra Amarilla el crecimiento es sostenido y cada año se han puesto en cultivo unas 150 ha nuevas. 
Estos perfiles temporales reflejan, en parte, un patrón espacial de ocupación del suelo, desde las zonas más aptas y próximas al fondo del valle hacia las más alejadas. En Copiapó parece que se ha puesto en cultivo prácticamente toda la superficie económicamente utilizable con la tecnología y costes de explotación actuales; en Tierra Amarilla se ralentiza también el ritmo de creación de nuevas plantaciones de vides. Es bastante probable que en unos cuantos años se estabilice la superficie dedicada a viñedos para uva de mesa, puesto que en los terrenos libres es más costosa su explotación y la disponibilidad de agua es cada día más escasa.

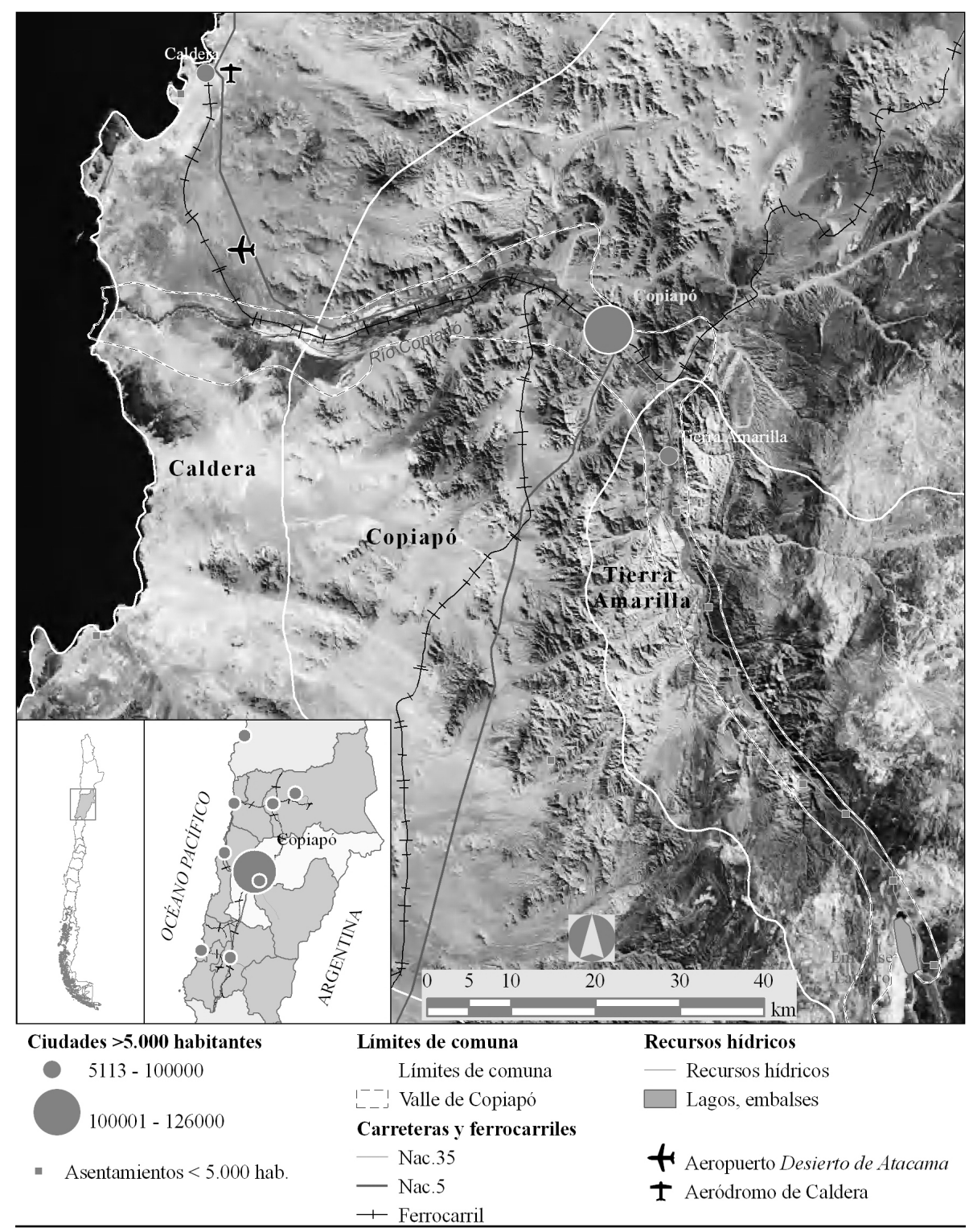

Fuente: Elaboración propia en base a @ 2009 ESRI, i-cubed, GeoEye, World Imagery. 


\section{Cuadro $\mathrm{N}^{\circ} 1$}

Evolución de la superficie de viñedo según su variedad en la Región de Atacama,

Copiapó y Tierra Amarilla (1994-2006)

\begin{tabular}{|c|c|c|c|c|c|c|}
\hline \multirow{2}{*}{ Año } & \multicolumn{3}{|c|}{ Región de Atacama } & \multicolumn{3}{c|}{ Uva de mesa (ha) } \\
\cline { 2 - 6 } & \multicolumn{3}{|c|}{ Variedad (ha) } \\
\cline { 2 - 6 } & Pisqueras & De mesa & Total & Copiapó & Tierra Amarilla & Total \\
\hline 1994 & 724,7 & $5.571,7$ & $6.296,4$ & $1.098,6$ & $4.071,9$ & $5.170,5$ \\
1995 & 731,1 & $5.959,5$ & $6.690,6$ & $1.021,3$ & $4.526,1$ & $5.547,4$ \\
1996 & 746,1 & $6.240,5$ & $6.986,6$ & $1.009,8$ & $4.768,9$ & $5.778,7$ \\
1997 & 781,0 & $6.342,0$ & $7.123,0$ & $1.027,0$ & $4.775,0$ & $5.802,0$ \\
1998 & 793,0 & $6.614,0$ & $7.407,0$ & $1.051,0$ & $4.985,0$ & $6.036,0$ \\
1999 & 798,0 & $6.879,0$ & $7.677,0$ & $1.013,0$ & $5.193,0$ & $6.206,0$ \\
2000 & 797,4 & $6.912,9$ & $7.710,3$ & 984,2 & $5.254,8$ & $6.239,0$ \\
2001 & 647,0 & $7.357,9$ & $8.004,9$ & 994,0 & $5.607,2$ & $6.601,2$ \\
2002 & 619,5 & $7.564,4$ & $8.183,9$ & $1.012,8$ & $5.703,0$ & $6.715,8$ \\
2003 & 614,8 & $7.691,1$ & $8.305,9$ & $1.001,4$ & $5.838,1$ & $6.839,5$ \\
2004 & 601,0 & $8.143,0$ & $8.740,0$ & $1.016,0$ & $6.209,0$ & $7.225,0$ \\
2005 & 655,0 & $8.737,0$ & $9.392,0$ & $1.215,0$ & $6.435,0$ & $7.650,0$ \\
2006 & 657,0 & $8.540,0$ & $9.197,0$ & $1.102,0$ & $6.399,0$ & $7.501,0$ \\
\hline
\end{tabular}

Fuente: Elaboración propia en base a ODEPA.

Figura $\mathrm{N}^{\circ} 2$

Evolución de la superficie de viñedo en la Región de Atacama y en el valle de Copiapó (1994-2006)

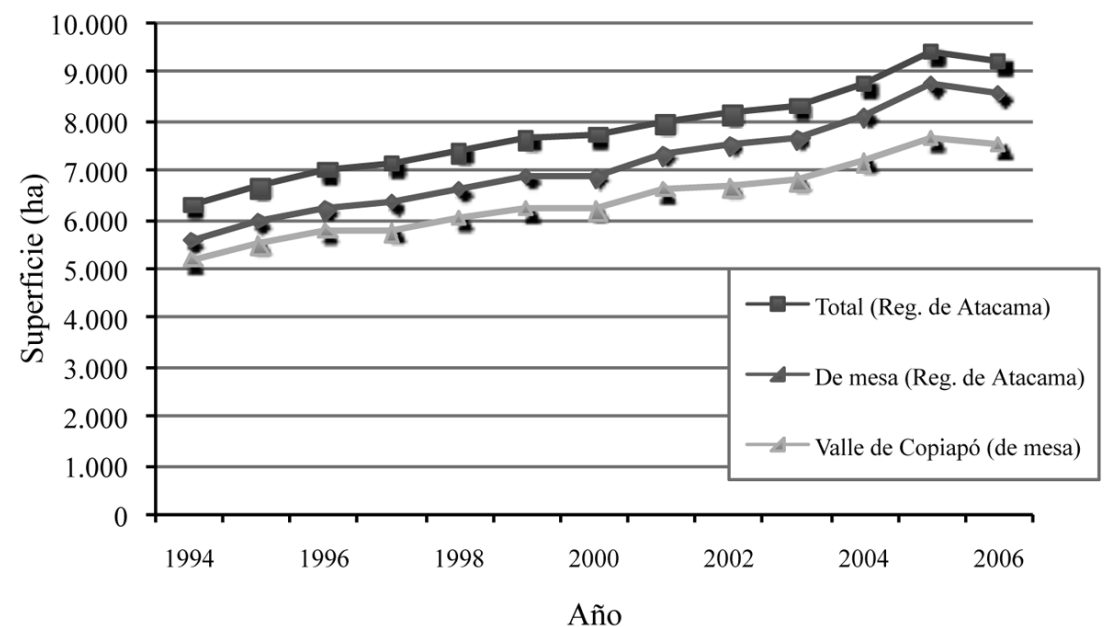

Fuente: Elaboración propia en base a catastro de vides 1994-2003, ODEPA; catastro Vitícola Nacional, SAG-INE. 


\section{Explotaciones de gran tamaño y muy tecnificadas}

El minifundismo agrario junto con la presencia de grandes latifundios constituye uno de los rasgos definitorios de la estructura regional de las explotaciones agrarias. Solo el $8 \%$ de las explotaciones se clasifican como grandes (mayores de $50 \mathrm{ha}$ ), aunque comprenden el 99,7\% de la superficie cultivada, si bien se incluyen también grandes extensiones propiedad de las instituciones (Cuadro $\mathrm{N}^{\circ}$ 2). Los fundos vitícolas superan con frecuencia las 100 ha y algunos alcanzan las 500 y 800 ha. También las parcelas de estas últimas suelen tener una extensión considerable, y no es raro que superen las 100 ha; algunas explotaciones tienen 500 ha de viñedo en una sola pieza. La puesta en cultivo de tierras baldías ha sido un mecanismo habitual de ampliación de superficie de las grandes explotaciones, aunque esta apropiación de terrenos colonizados ha generado también tensiones con la población local.

Además de la superficie, la dimensión económica de las explotaciones es también considerable, entre otras razones por la gran intensidad de capital invertido para la tecnificación de los procesos de producción y comercialización (investigación agraria, manejo de los cultivos y de los suelos, prospección de mercados). Estas inversiones no son asequibles a los presupuestos de la agricultura familiar, que tan solo significa el $1 \%$ de la superficie regional dedicada a producción de uva de mesa, sino a las grandes empresas y grupos inversores, principalmente chilenos pero extrarregionales (Cuadro $\mathrm{N}^{\circ} 2$ ).

En general, en las explotaciones dedicadas a la producción de uva para la exportación, se siguen métodos científicos de organización y de producción, y se utilizan tecnologías avanzadas en todos los procesos, lo que constituye una de las claves de su elevada productividad: unos $25.000 \mathrm{~kg} / \mathrm{ha}$ y hasta $30.000 \mathrm{~kg} / \mathrm{ha}$ en algunas variedades y suelos.

Otra de las razones de la reconocida competitividad de estas producciones se encuentra en la disponibilidad de mano de obra cualificada.

Junto a los empleados fijos, algunos profesionales muy especializados, se emplean trabajadores estacionales que en los períodos de mayor afluencia (noviembre-enero) pueden rebasar los 16.000 temporeros en el valle de Copiapó. En la investigación llevada a cabo por el Centro de Estudios para el Desarrollo de la Mujer (CEDEM, 2005) se describen de forma exhaustiva los aspectos fundamentales del mercado de trabajo en este valle.

\section{Cuadro $\mathrm{N}^{\circ} 2$}

Estructura de las explotaciones agrarias con tierras de la Región de Atacama según su superficie y condición jurídica (2007)

\begin{tabular}{|c|c|c|c|c|c|c|}
\hline \multirow[b]{2}{*}{$\begin{array}{l}\text { Superficie } \\
\text { (ha) }\end{array}$} & \multirow[b]{2}{*}{ Informantes } & \multicolumn{2}{|c|}{ Superficie } & \multicolumn{3}{|c|}{ Condición jurídica de los productores } \\
\hline & & $\mathrm{Ha}$ & $\%$ & & Informantes & $\begin{array}{l}\mathrm{Ha} \\
(000)\end{array}$ \\
\hline$<1$ & 680 & 351,29 & 0,01 & Explotaciones con tierra & 2.880 & 3.770 \\
\hline $1 \mathrm{a}<5$ & 1.226 & $2.721,37$ & 0,07 & Personas naturales & 2.704 & 817 \\
\hline $5 a<50$ & 571 & $8.681,92$ & 0,23 & ictores i & 2.497 & 500 \\
\hline $50 \mathrm{a}<100$ & 58 & $3.947,60$ & 0,10 & Personas jurídicas & 176 & 2.953 \\
\hline $100 \mathrm{a}<500$ & 73 & $16.924,1$ & 0,45 & - Sociedades anónimas y & 151 & 829 \\
\hline$>500$ & 82 & $3.737 .651,9$ & 99,13 & - Instituciones & 14 & 1.678 \\
\hline
\end{tabular}

Fuente: Elaboración propia en base a INE (2007). 


\section{Diversificación funcional y servicios a los productores agrarios}

La base económica, la trayectoria demográfica y las funciones de Copiapó han estado históricamente unidas a la marcha de la minería. Desde mediados de los ochenta del pasado siglo se han añadido otras actividades, como la agraria y los servicios, que contribuyen a la diversificación de la economía, de las funciones urbanas y de la composición social, e inducen otros cambios paisajísticos $y$ territoriales.

El desenvolvimiento combinado de estos factores, junto con la acción de las instituciones locales, regionales y del Estado, y de la sociedad local, ha promovido a Copiapó a los puestos superiores de la jerarquía urbana chilena. Esta ciudad es el nodo que enlaza los sistemas locales de la región, entre sí y con el nivel nacional $y$, en cierta medida, con el mundo.

Una condición fundamental para el buen desempeño de funciones urbanas especializadas radica en la existencia de buenas infraestructuras de transporte y comunicaciones, que garanticen las conexiones intrarregionales, con el resto del país y del mundo. En lo que se refiere a las carreteras, la ruta 5, verdadera columna vertebral de la red, articula en sentido norte-sur las principales ciudades y capitales de Chile, entre ellas Caldera, Copiapó, Vallenar y Huasco en la Región de Atacama, y La Serena en la vecina Región de Coquimbo. La red intrarregional está formada por ejes transversales trazados por los fondos de los valles principales desde los Andes hacia la costa para enlazar con la ruta 5, como la carretera C-35 que recorre el valle de Copiapó, y por otras vías secundarias que conectan los valles entre sí. Los ferrocarriles transportan principalmente los productos de la minería hasta los puertos.

El transporte de pasajeros a larga distancia se realiza a través del nuevo aeropuerto Desierto de Atacama, que entró en servicio en 2005 y se halla situado al pie de la ruta 5, $50 \mathrm{~km}$ al noroeste de Copiapó y a $25 \mathrm{~km}$ de Caldera. Los vuelos tienen origen y destino en Santiago, que es el distribuidor hacia el resto de aeropuertos. Ello no obstante, han funcionado otras relaciones con aeropuertos regionales, como el de Iquique, Antofagasta, Calama, La Serena, y se estudia establecer servicios con otros aeropuertos de esta categoría.

\section{Especialización funcional y rango en la jerarquía urbana}

El crecimiento demográfico de Copiapó es más destacable si se tiene en cuenta que se produce en una región de escaso peso demográfico respecto de Chile, y de valores bajos en términos absolutos (Cuadro $\mathrm{N}^{\circ} 3$ ).

El crecimiento de la población regional ha sido, en general, moderado y con tasas similares o ligeramente superiores al promedio nacional. Las tasas de crecimiento más elevadas se registran en los decenios 1960-1970 y en 1982-1992, y se explican por el florecimiento de la minería y la atracción de los flujos migratorios campo-ciudad; desde mediados de los 80, el despegue y consolidación de las actividades agrarias orientadas a la exportación también atraen y fijan población, especialmente en el valle de Copiapó. Sin embargo, la tasa neta migratoria regional es negativa en el quinquenio 1997-2002 (ViIlalón et al., 2007).

En cambio, el aumento de la población de Copiapó en los últimos 50 años se puede calificar de espectacular, hasta el punto de que en 2002 su censo de población triplicaba con creces al de 1960. Entre 1997 y 2002 la comuna de Copiapó recibió 16.752 inmigrantes, de los que un tercio (5.527) procedían de comunas de la Región de Atacama y los dos tercios restantes llegaron de las demás regiones del país. Al contrario, en otras comunas de la región, el contingente demográfico se ha estancado o ha retrocedido (Chañaral, Huasco y Diego de Almagro4).

Las transformaciones recientes de la sociedad y economías chilenas se manifiestan,

\footnotetext{
4 Por ejemplo, entre 1997 y 1999 se desmanteló un poblado minero en esta comuna, parte de cuya población, unas 1.360 personas, se trasladaron a Copiapó y a La Serena (Pontificia Universidad Católica de Chile, 2000)
} 
de forma acusada, en los cambios de las funciones urbanas promovidos por el avance imparable de funciones características de las sociedades de los servicios y de la información.

Este proceso de transición del modelo funcional urbano es muy complejo, ya que en él interactúan diversos factores a veces de modo contradictorio. Sus propiedades principales se resumen en estas tres tendencias: 1) el retroceso relativo de las actividades de producción, especialmente intenso en determinados tipos, aunque las funciones productivas todavía mantienen un peso elevado en las economías urbanas; 2) el crecimiento de la producción inmaterial y de los servicios, como consecuencia de la externalización de tareas desde las empresas de producción, de la incorporación de información en los nuevos métodos de organización y fabricación, y por la difusión de nuevos estilos de consumo; 3 ) el estado desigual del proceso en las ciudades, principalmente en razón de su tamaño, de su localización geográfica y de su posición en el sistema urbano.

En el Cuadro $\mathrm{N}^{\circ} 4$ se recogen los Coeficientes de localización (QL) por esferas y ramas de actividad para Copiapó, Gran Santiago, y 21 áreas urbanas entre 100 mil y 500 mil habitantes, calculados a partir de la población ocupada en 2002. Los valores evidencian, por una parte, las distintas fases del proceso de transición funcional en que se hallan las ciudades y, por otra, el trabajo que desarrollan en el sistema urbano.
La composición funcional más avanzada es la del Gran Santiago. En efecto, aquí se localizan con más intensidad las funciones de producción inmaterial y de servicios y, en menor medida, las de producción de bienes. No obstante, también reviste gran importancia la producción industrial, cuyo QL es mayor que en cualquier otro grupo de ciudades. También se asientan aquí prácticamente todos los órganos y organizaciones extraterritoriales y otros servicios e instituciones, que traducen un modelo funcional propio de su indiscutible papel de metrópoli nacional y de una ciudad de clase mundial.

El grupo intermedio está compuesto por 21 áreas urbanas entre $100 \mathrm{mil}$ y $500 \mathrm{mil}$ habitantes, entre las que se encuentran las capitales regionales, excepto una, y otras ciudades. Su estructura funcional es bien distinta de la del Gran Santiago, y se caracteriza, sobre todo, por su elevada especialización en las ramas productivas de minería y pesca que, en solitario o con otras, constituyen la base económica principal de algunas ciudades de este grupo. La elevada especialización en la rama de administración pública es un trasunto fiel de las funciones administrativas propias de las capitales regionales.

El perfil funcional de Copiapó, a su vez, difiere en algunos aspectos significativos del correspondiente a la categoría de ciudades de la que forma parte. En efecto, en relación con esta, en Copiapó sobresalen los QL de las ramas minas, agricultura y actividades inmo-

Cuadro $\mathrm{N}^{\circ} 3$

Evolución de la población de la Región de Atacama y de la comuna de Copiapó (1960-2002)

\begin{tabular}{|c|c|c|c|c|c|c|}
\hline \multirow{2}{*}{ Censo } & \multicolumn{2}{|c|}{ Región de Atacama } & \multicolumn{2}{c|}{ Comuna de Copiapó } & \multicolumn{2}{c|}{ Ciudad de Copiapó } \\
\cline { 2 - 7 } & Población & $\begin{array}{c}\text { Atacama / } \\
\text { Chile (\%) }\end{array}$ & Población & $\begin{array}{c}\text { Copiapó/ } \\
\text { Atacama (\%) }\end{array}$ & Población & $\begin{array}{c}\text { Copiapó/ } \\
\text { Comuna (\%) }\end{array}$ \\
\hline 1960 & 115.235 & 1,56 & 38.371 & 33,30 & 30.123 & 78,50 \\
1970 & 153.888 & 1,73 & 51.731 & 27,61 & 45.194 & 87,36 \\
1982 & 183.407 & 1,62 & 71.612 & 39,05 & 69.045 & 96.42 \\
1992 & 230.873 & 1,73 & 100.907 & 43,71 & 98.188 & 97,31 \\
2002 & 254.336 & 1,68 & 129.091 & 50,76 & 125.983 & 97,59 \\
\hline
\end{tabular}

Fuente: Elaboración propia en base a censos de población, INE. 
biliarias y empresariales, lo que confirma una de las hipótesis de este trabajo: la asociación espacial entre actividades productivas y ciertos servicios a la producción. Sin embargo, las empresas más especializadas de servicios avanzados para la producción agraria se ubican, preferentemente, en Santiago.

Con el detalle proporcionado por las 60 categorías de ocupación de la variable giro del Censo de Población y Vivienda de 2002, se puede precisar más la especialización funcional de Copiapó: los valores más elevados del QL, próximo a 8, corresponden a las actividades relacionadas con la extracción de minerales (actividades del Censo: 6, 7 y 8), seguidos de la agricultura, ganadería y servicios conexos con un QL de 2,02 (actividad 1 del Censo). La categoría alquiler de maquinaria (actividad 48) presenta también cierto grado de especialización $(\mathrm{QL}=1,9)$, así como captación, depuración y distribución de agua $(\mathrm{QL}=1,4)$, fabricación de maquinaria, transporte por vía terrestre y administración pública $(\mathrm{QL}=1,2)$.

La distribución rango-tamaño de las ciudades chilenas refleja la adaptación del sistema urbano nacional a las condiciones que determinan la localización interurbana de la población, en especial a la mejora de los transportes, a la distribución del empleo

\section{Cuadro $\mathrm{N}^{\circ} 4$}

Coeficientes de localización por esferas y ramas de actividad calculados para las ciudades mayores de 5.000 habitantes (2002)

\begin{tabular}{|l|c|c|c|}
\hline \multicolumn{1}{|c|}{$\begin{array}{c}\text { Esfera } \\
\text { Rama }\end{array}$} & Copiapó & $\begin{array}{c}\text { (21) Áreas urbanas } \\
(100-500 \text { miles hab. })\end{array}$ & Gran Santiago \\
\hline Producción & 1,17 & 0,94 & 0,86 \\
A. Agricultura & 1,75 & 0,66 & 0,28 \\
B. Pesca & 0,21 & 1,74 & 0,06 \\
C. Minas & 6,82 & 2,51 & 0,27 \\
D. Industria manufacturera & 0,59 & 0,82 & 1,13 \\
E. Construcción & 1,14 & 1,16 & 0,86 \\
Intermediación & 0,97 & 1,01 & 1,07 \\
F. Energía & 1,06 & 1,12 & 0,92 \\
G. Comercio & 0,97 & 1,08 & 1,01 \\
H. Hoteles & 0,88 & 0,98 & 1,24 \\
I. Transporte & 0,90 & 1,12 & 1,03 \\
J. Finanzas & 0,71 & 1,09 & 0,96 \\
K. Actividades inmobiliarias y empresariales & 1,02 & 0,82 & 1,40 \\
Reproducción social & 0,91 & 1,04 & 1,03 \\
L. Administración pública & 1,12 & 1,23 & 0,86 \\
M. Enseñanza & 1,09 & 1,17 & 0,91 \\
N. Salud & 0,78 & 1,01 & 1,12 \\
O. Servicios sociales & 0,84 & 1,02 & 1,11 \\
P. Servicio doméstico & 0,72 & 0,92 & 1,15 \\
Q. Órganos extraterritoriales & 0,05 & 0,24 & 1,95 \\
\hline
\end{tabular}

Fuente: Escolano y Ortiz, 2007. 
y a los nuevos modelos de urbanización metropolitana. En general, entre 1972 y 2002 se reduce la primacía del sistema, debido al crecimiento de las grandes ciudades y de las ciudades medias, y a la estabilidad demográfica reciente del Gran Santiago.

Sin embargo, la persistencia estructural del sistema es compatible con intensos cambios a escala local. Copiapó es una de las ciudades que desde 1960 asciende constantemente en la jerarquía urbana chilena: en cada período censal ha escalado un rango desde entonces.

Para este trabajo es importante subrayar que la intensidad y resultados de la competencia urbana parece que se relacionan positivamente y en gran medida con el grado de internacionalización de sus economías: la mayor parte de las ciudades que avanzan en sus rangos, o permanecen en los que ya tenían, son aquellas cuya producción se dirige a mercados internacionales (Escolano et al., 2007).

\section{Los servicios a los productores agrarios}

El desarrollo de la minería y de la agricultura orientadas a la exportación ha generado un modelo socioterritorial específico, uno de cuyos ejemplos más característicos lo constituye el valle de Copiapó. Junto a las modalidades generales de organización socioeconómica, se ha creado un sistema técnico de considerable magnitud, compuesto por instalaciones físicas (carreteras y caminos, fábricas, redes de riego, almacenes, depósitos frigoríficos, instalaciones portuarias, entre otros), y por actividades de servicio necesarias para organizar todas las tareas productivas.

Las actividades directas e indirectas relacionadas con la producción y exportación de cobre y sus derivados y de uva fresca, han creado puestos de trabajo fijos y temporales que forman la base económica de Copiapó. Por tanto, la evolución demográfica, el mercado de trabajo, las relaciones sociales, e incluso el crecimiento urbano, dependen, en gran medida, de la marcha de aquellos sectores económicos.

Los impactos en el territorio no han sido menores. El paisaje refleja las tremendas heridas de la minería, y los espectaculares efectos cromáticos de las vides que reverdecen o amarillean las laderas semidesérticas del valle. Ambas actividades han deteriorado los recursos hídricos y han desencadenado o perturbado otros procesos geomorfológicos y geoquímicos.

Por otro lado, la organización espacial de las actividades económicas y el rango de capital regional han acentuado la diversificación funcional y el carácter de lugar central de Copiapó: aquí se concentran las empresas de servicio, los profesionales, los establecimientos financieros y los de seguros, los centros docentes, los hoteles y otras funciones urbanas que hacen de esta ciudad uno de los nodos de la red urbana chilena. En el resto de los asentamientos, excepto Caldera, predomina la función residencial acompañada de algunos comercios y servicios básicos a la población; además, todos ellos están especializados en actividades productivas.

Antes de analizar los servicios a los productores agrarios, es necesario advertir que resulta difícil identificar este tipo de empresas de servicio, ya que su actividad, salvo casos específicos, se dirige tanto a los productores agrarios como a los de otras ramas productivas. En función de la descripción recogida en el registro de patentes y de los datos del trabajo de campo, se han incluido aquí aquellas empresas que prestan servicios a los productores agrarios en grado significativo.

En relación con los objetivos de este trabajo, es importante diferenciar las empresas según su mayor o menor dependencia funcional con las unidades productivas, y por su nivel de especialización; estos criterios se correlacionan con la proximidad espacial (o lejanía) de las empresas de servicios con las unidades de producción. La correcta caracterización de las empresas de servicios según la demanda a la que atienden y el modo de prestar el servicio, es imprescindible para comprender el desarrollo de los servicios a la producción (Martinelli, 1991a). En general, es útil la distinción entre servicios comerciales y las actividades financieras, de seguros e inmobiliarias.

Las empresas que proporcionan bienes o servicios a los productores relacionados con 
equipamientos e instalaciones físicas de uso continuado, mantienen estrechos vínculos funcionales con las unidades de producción, lo que suele manifestarse también en su gran proximidad espacial. Esta asociación es evidente en el caso de la venta de cierta maquinaria agrícola y de sus repuestos, cuyas dependencias ocupan abundantes naves de los polígonos industriales al oeste de Copiapó (por ejemplo, Salinas y Fabres en el sector EI Mirador), en los márgenes de la ruta 5. Aunque en el registro de patentes no se especifica, también son abundantes los servicios de reparación de alquiler de máquinas para la agricultura, cuyo negocio va en alza (Cuadro № 5 ).

Algunos servicios prestados por profesionales (ingenieros agrónomos, enólogos) y por empresas (agencias de aduanas) guardan también relación directa con la viticultura. Cabe añadir que la enseñanza universitaria pública no imparte titulaciones superiores especializadas en los dominios de la agronomía.
El resto de servicios a los productores tienen un rango de clientes más amplio aunque, como se ha dicho, la especialización de Copiapó en estas actividades es más elevada que en las ciudades de su grupo, que incluso comprende núcleos de mayor tamaño en los que, a priori, la presencia de esta clase de servicios es más notoria. Los servicios más importantes son los de asesoría a empresas y la banca, finanzas y seguros. Los primeros suelen identificarse de pequeñas empresas locales, mientras que los otros dos tipos son frecuentes en las filiales de grandes grupos empresariales.

Finalmente, también es significativa la presencia de servicios de contabilidad y auditoría, procesamiento de datos y comunicaciones. Se trata asimismo de empresas individuales, de pequeño tamaño, salvo las de comunicaciones que se corresponden con centros locales y regionales de grandes empresas públicas o privadas del sector.

\section{Cuadro $\mathrm{N}^{\circ} 5$}

Patentes y trabajadores de las empresas de servicios relacionadas con la agricultura en Copiapó (2000 y 2007)

\begin{tabular}{|l|r|r|r|r|}
\hline \multirow{2}{*}{ Giro } & \multicolumn{2}{|c|}{ Patentes } & \multicolumn{2}{c|}{ Trabajadores } \\
\cline { 2 - 5 } & 2000 & 2007 & 2000 & 2007 \\
\hline Alquiler de maquinaria & 3 & 6 & 144 & 160 \\
Ingenieros agrónomos & 1 & 1 & 1 & 1 \\
Agentes de aduanas & 1 & 1 & 15 & 15 \\
Asesoría tributaria & 1 & 1 & 1 & 1 \\
Asesoría económico-financiera & 4 & 13 & 8 & 64 \\
Bancos & 13 & 20 & 186 & 231 \\
Financieras & 4 & 5 & 73 & 73 \\
Otros servicios financieros & 1 & 3 & 1 & 14 \\
Compañías de seguros & 6 & 8 & 46 & 50 \\
Agentes de seguros & 5 & 4 & 9 & 7 \\
Servicios de contabilidad y auditoría & 52 & 59 & 61 & 64 \\
Proceso de datos & 5 & 6 & 23 & 26 \\
Servicios de publicidad & 2 & 2 & 2 & 2 \\
Comunicaciones & 10 & 43 & 80 & 141 \\
Total general & 108 & 172 & 650 & 849 \\
\hline
\end{tabular}

Fuente: Ilustre Municipalidad de Copiapó, 2008. 
Figura $\mathrm{N}^{\circ} 3$

Evolución de las patentes y trabajadores de los servicios a los productores agrarios en Copiapó (2000 y 2007)

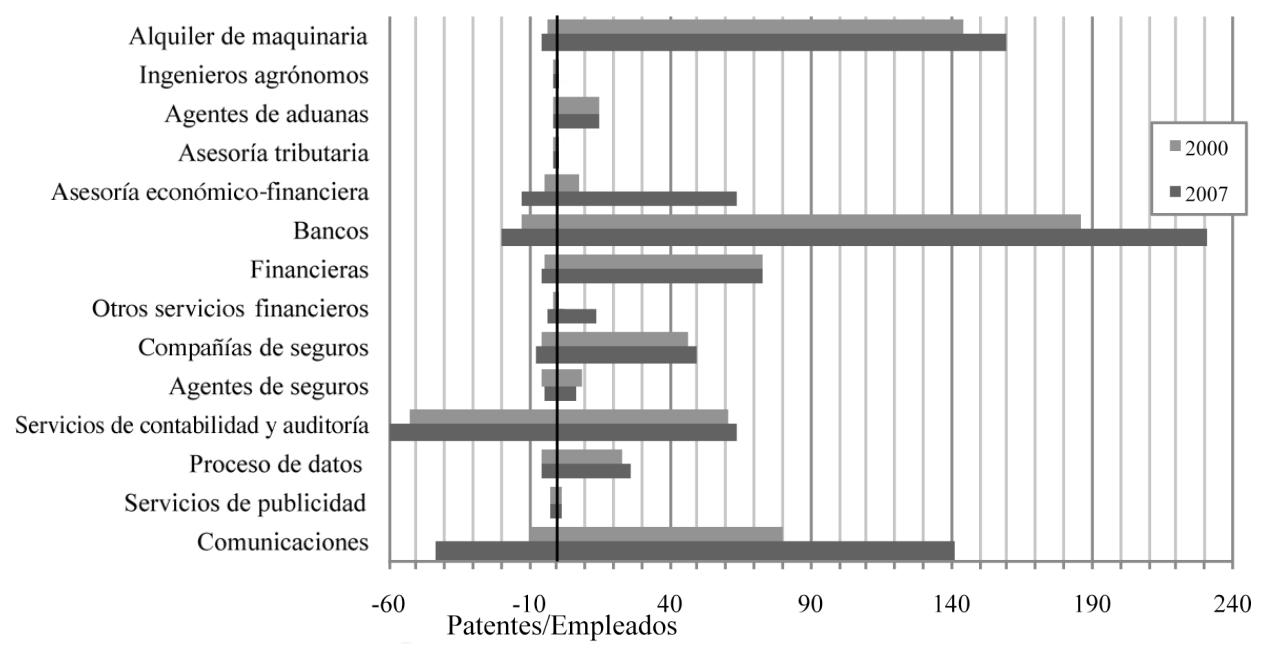

Fuente: Elaboración propia en base a datos Ilustre Municipalidad de Copiapó, 2008.

En lo que respecta a la actividad agraria, ciertos bienes, especialmente algunos tipos de maquinaria y algunos servicios especializados, se obtienen en la ciudad de La Serena. En Santiago residen algunos profesionales que trabajan en fundos de Copiapó, y allí se localizan las sedes de las empresas propietarias de las mayores explotaciones agrarias del valle y las que prestan los servicios más especializados al sector (investigación, prospección de mercados, formación especializada, aseguradoras, entre otros).

\section{Consideraciones finales}

La fuerte especialización de algunas economías regionales y locales en actividades orientadas a la exportación de materias primas minerales y sus derivados o productos agrícolas, va acompañada también del desarrollo de los servicios para las empresas, pero en grado moderado, no en proporción directa a la intensidad productiva.

La producción de cobre y derivados y de uva fresca que se venden en mercados exteriores, constituye la base económica de Copiapó. Además, la magnitud física (y también la económica) de esta producción es consi- derable (valor de la exportación de uva de mesa de la Región de Atacama: 165.947.517 dólares FOB). Aunque aquí los servicios a las empresas alcanzan un nivel de especialización superior al de otras ciudades de tamaño demográfico similar, se trata, en general, de servicios de tipo comercial, prestados por pequeñas empresas o microempresas locales, o bien por filiales de grandes corporaciones. Las empresas potentes de servicios avanzados a la producción apenas están representadas.

Además de la mundialización de los mercados, las grandes empresas de producción y servicios adoptan también nuevas formas de organización y de difusión internacional. La formación de redes de empresas y otros factores han propiciado renovadas modalidades de la actuación de las tendencias a la concentración-dispersión espacial de los centros de decisión y de las unidades de gestión y de administración. Las grandes metrópolis son los nodos de las nuevas formas espaciales de la organización de la producción a escala mundial. En Chile, Santiago desempeña esta función y aquí se localizan las principales empresas de servicios a los productores y prácticamente todas de servicios avanzados para la producción. La 
dimensión y funciones económicas y otros aspectos sociales y territoriales que adquiere la organización social y productiva a escala regional y local se definen en relación a este marco global.

\section{Referencias bibliográficas}

ALBRECHTS, L.; MOULAERT, F.; ROBERTS, P. \& SWYNGEDOUW, E. (eds.) Regional policy at the crossroads. European perspectives. London: j. Kingsley \& Regional Studies Associationed, 1989.

ARNADE, C. \& SPARKS, A. Chile's agricultural diversification. Agricultural Economics, 1993, vol. 9, No 1, p. 1-13.

GOBIERNO REGIONAL DE ATACAMA. Bases para el Plan Regional de Gobierno 2006-2010. Santiago: Gobierno Regional de Atacama, Gobierno de Chile, 2005.

BAILLY, A. S. Le rôle des activités de service dans le développement régional sisse. Annales de géographie, 1985, vol. 523, p. 257-269.

BAILLY, A. S. \& COFFEY, W. J. Localisation des services à la production et resturations économiques. Un analyse théorique. L'Espace Géographique, 1994, vol. 1, p. 224-230.

BAILLY, A. S. y MAILLAT, D. Servicios a las empresas y desarrollo regional. Ekonomiaz, 1989, vol. 13-14, p. 128-137.

BARCET, A.; BONAMY, J. \& MAYERE, A. Les services aux entreprises: problèmes théoriques et méthodologiques. Recherches économiques et sociales. La documentation française, 1984, vol. 9, p. 254-277.

BARTON, J. R.; Gwynne, R. N. \& Murray, W. E. Competition and co-operation in the semiperiphery: closer economic partnership and sectoral transformations in Chile and New Zealand. The Geographical Journal, 2007, vol. 173, No3, p. 224-241.

BEAVERSTOCK, J. V.; SMITH, R. G. \& TAYLOR, P. J. A roster of world cities. Cities, 1999, vol. 16, Nº 6, p. 445-458.
BECKOUCHE, P. \& DAMETTE, F. Une griIle d'analyse globale de l'emploi. Economie \& Statistiques, 1993, vol. 270, p. 37-50.

BENKO, G. y LIPIETZ, A. (eds.) Las regiones que ganan: distritos y redes. Los nuevos paradigmas de la geografía económica. Valencia: Institución Alfons el Magnànim, 1994.

CENTRO DE ESTUDIOS PARA EL DESARROLLO DE LA MUJER (CEDEM). Empleo y condiciones de trabajo en la producción de uva de exportación en el valle de Copiapó. Copiapó: Centro de Estudios para el DesarroIlo de la Mujer, FNDR III Región, 2005.

COFFEY, W. J. \& BAILlY, A. S. Producer services and systems of flexible production. Urban Studies, 1992, vol. 29, Nº 6, p. 857 868.

COFFEY, W. J. \& POLESE, M. Trade and location of producer services: a canadian perspective. Environment and Plannig A, 1987, vol. 19, No 5, p. 597-612.

DANIELS, P. W. Services industries. A geographical appraisal. London: Methuen, 1985.

DANIELS, P. (ed.) Services and metropolitan development: international perspectives. Londres: Routledgeed. 1991.

DANIELS, P. (ed.) The geography of services. Londres: Frank Cassed, 1993.

DANIELS, P. W. \& MOULAERT, F. (eds.) The changing geography of advanced producer services. Theoretical and empirical perspectives. London: Belhaven Press, 1991.

DE MATTOS, C.; DUCCI, M. E.; RODRÍGUEZ, A. y YÁÑEZ, G. (eds.) Santiago en la globalización: ¿una nueva ciudad? Santiago: SUR Corporación de Estudios Sociales y Educación; Instituto de Estudios Urbanos y Territoriales, Pontificia Universidad Católica de Chile, 2004.

ESCOLANO, S.; ORTIZ, J. y MORENO, R. Globalización y cambios funcionales recientes en las ciudades del sistema urbano chileno. Cuadernos Geográficos, 2007, vol. 41, No 2 , p. 33-60. 
GERSHUNY, J. y MILES, I. La nueva economía de los servicios. La transformación del empleo en las sociedades industriales. Madrid: Ministerio de Trabajo y Seguridad Social, 1988.

GONZÁLEZ, D. y RODRÍGUEZ, J. Redistribución espacial y migración interna de la población en Chile en los últimos 35 años (1965-2002): una síntesis de las hipótesis y la evidencia. Estudios Demográficos y Urbanos, 2006, vol. 21, No 202, p. 369-404.

GWYNNE, R. N. Direct foreign investment and non-traditional export growth in Chile: the case of the forestry sector. Bulletin of Latin American Research, 1996, vol. 15, $\mathrm{N}^{\circ} 3$, p. 341-357.

GWYNNE, R. N. Globalisation: commodity chains and fruit exporting regions in Chile. Tijdschrift voor Economische en Sociale Geographie, 1999, vol. 90, No 2, p. 211-225.

ILLERIS, S. Bibliography of the role of services in regional development. Bruselas: Commision for the European Comunities, FAST Ocassional Paper 67, 1985.

ILLERIS, S. Services and Regions in Europe. Aldershot: Gower, 1989.

ILUSTRE MUNICIPALIDAD DE COPIAPÓ. Registro de patentes comerciales. Copiapó: Ilustre Municipalidad de Copiapó, 2008.

INSTITUTO NACIONAL DE ESTADÍSTICAS (INE). Censos de población y viviendas (varios años). Santiago: INE, 2002.

INSTITUTO NACIONAL DE ESTADÍSTICAS (INE). Censo agropecuario de 2007. Santiago: INE, 2007.

INSTITUTO NACIONAL DE ESTADÍSTICAS (INE). Compendio estadístico año 2008. Santiago: INE, 2009. Disponible en Internet: http://www.ine.cl/canales/menu/publicaciones/compendio_estadistico/compendio_estadistico2008.php

MARTINELLI, F. A demand-oriented approach to understanding producer services. In: DANIELS, P. W. \& MOULAERT, F. (ed.). The changing geography of advanced producer services: theoretical and empirical perspectives. Londres: Belhaven Press, 1991a, p. 15-29.

MARTINELLI, F. Producer services location and regional development. In: DANIELS, P. W. \& MOULAERT, F. (ed.). The changing geography of advanced producer services. Theoretical and empirical perspectives. London: Belhaven Press, 1991b, p. 15-29.

MONNOYER, M. C. \& PHILIPPE, J. Localisation factors adn development strategies of producer services. In: DANIELS, P. W. \& MOULAERT, F. (ed.). The changing geography of advanced producer services. Theoretical and empirical perspectives. London: Belhaven Press, 1991, p. 108-117.

MORENO, A. y ESCOLANO, S. El comercio y los servicios para la producción y el consumo. Madrid: Síntesis, 1992a.

MORENO, A. y ESCOLANO, S. Los servicios y el territorio. Madrid: Síntesis, $1992 \mathrm{~b}$.

MOULAERT, F. \& GALLOUJ, C. The locational geography of advanced producer service firms: the limits of economies of agglomeration. The Service Industries Journal, 1993, vol. 13, No 2, p. 91-106.

MOUlAERT, F. \& SCOTT, A. J. (eds.) Cities, enterprises and society on the eve of the 21 st century. Londres: Pintered. 1997.

MOULAERT, F.; SCOTT, A. J. \& FARCY, H. Producer services and the formation of urban space. In: MOULAERT, F. \& SCOTT, A. (ed.). Cities, enterprises and society on the eve of the 21st century. London: Pinter, 1997, p. 97-112.

NOYELLE, T. The shift to services, technological change and the restructuring of the system of cities in the Unites States. In: STÖHR, W. (ed.). International economic restructuring and the territorial community. Viena: U. N. Industrial Development Organization, 1986, p. 239-264.

ORGANIZACIÓN MUNDIAL DEL COMERCIO (OMC). Estadísticas de comercio Internacional. Ginebra: OMC, 2009. Disponible en Internet: http://www.wto.org/spanish/res_s/ statis_s/its2009_s/its09_toc_s.htm 
PARNREITER, C.; FISCHER, K. e IMHOF, $K$. El enlace faltante entre cadenas globales de producción y ciudades globales: el servicio financiero en Ciudad de México y Santiago de Chile. EURE, 2007, vol. 33, № 100, p. 135-148.

PONTIFICIA UNIVERSIDAD CATÓLICA DE CHILE. Potrerillos. Valorización y conservación del patrimonio arquitectónico. Santiago: Pontificia Universidad Católica de Chile, 2000.

RADA, J. F. Tecnología de la información y servicios. Ekonomiaz, 1989, vol. 13-14, p. 62-99.

RIFFO, L. y CALISTO, N. Crecimiento económico regional en Chile 1985-1998. Estadística y Economía, 1998, vol. segundo semestre, p. 51-74.

SASSEN, S. The global city: New York, London, Tokyo. Princeton: Princeton University Press, 1991.
SASSEN, S. Localizando ciudades en cuircuitos globales. EURE, 2003, vol. 29, № 88, p. 5-27.

SERPLAC. Plan de gobierno. Región de Atacama, 2006-2010. Santiago: SERPLAC Atacama, Gobierno de Chile, 2005.

SZARY, A. L. Regiones ganadoras y regiones perdedoras en el retorno de la democracia en Chile: poderes locales y desequilibrios territoriales. EURE, 1997, vol. 23, No 70, p. 59-78.

VELUT, S. Mondialisation et territoires de la régulation au Chili et en Argentine. Bulletin de l'Association de Géographes Françaises, 2005, vol. 4, p. 470-482.

VILLALÓN, G. FARIA, O. y ACUÑA, E. Migración interna regional 1997-2002. Santiago: INE, 2007. 